\title{
La investigación jurídica como un instrumento de mejoramiento del proceso enseñanza-aprendizaje del Derecho
}

\section{Legal research as a tool for improving the teaching-learning process of the law}

\author{
Gabriel Álvarez Undurraga ${ }^{1}$
}

\begin{abstract}
Resumen: la enseñanza del derecho es un área de la educación universitaria que se ha mantenido relativamente uniforme en el tiempo, a pesar de los cambios sociales y educativos que ha vivido la sociedad actual; cambios que se deben a la irrupción de los medios digitales y al cambio de paradigma con respecto a la relación profesor-alumno. El autor plantea la necesidad de que la enseñanza del derecho se modernice, para lo cual debe incorporar los cambios mencionados y, especialmente, considerar la investigación jurídica como una actividad permanente dentro del ámbito académico, y, además, colaborativa entre el docente y los estudiantes.
\end{abstract}

Palabras clave: enseñanza del derecho, investigación jurídica, cultura jurídica.

Abstract: legal education is an area of higher education that has remained relatively consistent over time, in spite of the social and educational changes that our society has lived: changes caused by the emergence of digital media and the transformation of the paradigm with respect to the teacher-student relationship. The author states the need for legal education to modernize, incorporate these changes and, especially, consider legal research as an ongoing activity within the academy sphere and as a collaborative process between teacher and students.

Key words: law teaching, legal research, legal culture.

\footnotetext{
${ }^{1}$ Abogado, graduado en la Universidad Central de Venezuela y profesor de Castellano y Literatura, graduado en la Universidad de Santiago de Chile. Ha realizado estudios de posgrado: Maestría en Literatura Latinoamericana en la Universidad Simón Bolívar, en la Universidad Central de Venezuela; especialización en Derecho del Trabajo, en la Universidad de los Andes de Venezuela; Metodología de la Investigación en Ciencias Sociales y Magíster en Derecho en la Facultad de Derecho de la Universidad de Chile. Es Doctor () en Estudios Americanos, Mención Relaciones Internacionales en la Universidad de Santiago de Chile. Ha sido profesor en Venezuela, en la Universidad Central de Venezuela, de la Universidad de los Andes, de la Universidad de Oriente y de la Universidad Metropolitana. Ha dictado cátedras en la Universidad de Atacama, Universidad Católica del Norte, Universidad de Talca, Universidad de los Andes, Universidad de Heidelberg, entre otras. Actualmente trabaja como profesor en la Facultad de Derecho de la Universidad de Chile, Universidad de Santiago, Academia de Guerra del Ejército de Chile, Universidad Central de Chile y en la Universidad San Sebastián. Correo electrónico: gabriel.alvarezu@ gmail.com.
} 


\section{Introducción}

Uno de los problemas más significativos en la educación moderna es el papel del profesor como parte integrante y protagonista de la práctica educativa. En este sentido, existen diversos enfoques que las teorías pedagógicas intentan resaltar y aplicar en modalidades del proceso enseñanza-aprendizaje, que el docente estaría en condiciones de llevar a cabo. Por otra parte, el cada vez más relevante e importante actor: el estudiante, quien está inserto en un contexto complejo y cambiante. Ambos son los protagonistas de la práctica educativa.

Otro aspecto destacado es la cultura participativa y colaborativa como eje de los procesos educativos, la que se ha ido modificando por la dinámica de este contexto.

Además, está la dinámica de los centros educativos, los cuales presentan problemas, pero que están atentos a los proyectos de mejoras en el ámbito educacional.

En la sociedad global que estamos viviendo, la educación continúa cumpliendo su papel de agente de socialización, pero ahora los sistemas educativos están apoyados por las nuevas tecnologías de la información, las cuales más que modernizar el proceso enseñanzaaprendizaje, utilizando los diversos instrumentos y aplicándolos a la situación específica que el docente pretende desarrollar, están generando una nueva forma de pensar y recrear este proceso, haciéndolo más crítico, creativo, propositivo y, por ende, reflexivo. Con las TIC a su alcance y la nueva perspectiva de alfabetización tecnológica multimodal e intercultural $^{2}$, el docente se enfrenta a una perspectiva de un saber más integral y totalizador, que estimule a los diversos actores de este sistema y genere la posibilidad real de transformarse en un líder o conductor, pero siempre con la participación de todos los agentes involucrados, entre ellos el alumno.

Los procesos enseñanza-aprendizaje deben considerar el nuevo contexto de la sociedad global y de la realidad chilena, en particular, para plantear innovaciones en la materia que permitan recrear este proceso con los diversos actores involucrados, entregando las posibilidades de un qué hacer más holístico y transformador, que incorpore a diversos sectores que antes no estaban considerados.

En los procesos educativos siempre se ha discutido el mejoramiento de la calidad de la enseñanza; la Educación Superior, a nivel universitario, no ha sido ajena a esta preocupación.

El proceso enseñanza-aprendizaje tradicional en la formación jurídica del futuro abogado actualmente está en crisis, debido a las transformaciones que demanda la sociedad, influida por los fenómenos de la globalización y la obsolescencia de los conocimientos. No es conveniente la enseñanza verticalista, basada sólo en la memorización de normas. En este contexto, resulta necesario estudiar el aporte que puede entregar la investigación jurídica en el proceso de formación de los futuros abogados. Por lo tanto, es indispensable generar una metodología de la investigación jurídica, que permita un cambio de la perspectiva dogmática y exegética tradicional que se utilizaba en Chile.

\footnotetext{
${ }^{2}$ BAUTISTA GARCÍA-VERA, Antonio. "Alfabetización tecnológica multimodal e intercultural”. En:
} Revista de Educación. 2007, núm. 143. 


\section{El papel del profesor}

La sociedad cuando crea una cultura, desea que ésta sea compartida por todos sus miembros, lo que se manifiesta en los contenidos que se transmiten obligatoriamente, con esto se pretende realizar un modo de convivencia que se considera bueno. En este sentido, la Educación Básica determina el verdadero nivel de vida, no sólo el económico; por lo tanto, la enseñanza obligatoria es educadora. Frente a esta situación, es necesario que el profesor de enseñanza obligatoria asuma un papel comprometido. Va a formar a todos los miembros de la sociedad a través de un sistema coactivo amparado por las leyes, teniendo que transmitir unos contenidos en cuya selección no ha formado parte. Es un funcionario del estado, pero debe ser, sentirse y comportarse como un funcionario de la sociedad. Un problema esencial es el proceso de formación del profesor, que debe ser científica y pedagógica, pero también con una conciencia social elevada.

Si repasamos la historia del profesorado, ésta se ha visto influida en la Edad Media por las trasmisión de dogmas culturales y religiosos; en la ilustración, su papel intentó ser liberador; actualmente ha pretendido ser formadora de personal cualificado que requiere el sistema productivo de una nación en vías de desarrollo. A su vez, la pedagogía crítica quiere hacer del profesorado un intelectual políticamente comprometido.

Siguiendo el pensamiento de Hargreaves ${ }^{3}$, las actuales pautas del cambio educativo están promovidas por la confrontación de dos fuerzas sociales: las de la modernidad y las de la postmodernidad. La modernidad, que arranca del pensamiento ilustrado, se define por el culto a la razón y a la ciencia, la confianza en la técnica para resolver el problema; defiende que hay una verdad común, una ética universal, una historia compartida por toda la humanidad. El postmodernismo, cree que la razón se ha desconfigurado por un afán de poder técnico, su eficacia ciega es equívoca, su confianza ha llevado al dogmatismo, al fanatismo, al etnocentrismo, al colonialismo y otras injusticias; piensan que hay que devolver a cada cultura su autonomía. El profesor Marina ${ }^{4}$ plantea que hay que superar este enfrentamiento, para ello propone la ultramodernidad, a la que define como una teoría de la inteligencia y de la creación estética. Señala que: "Los ultramodernos creemos que el trabajo de la inteligencia es a la vez más humilde y más trascendental. Su función es dirigir el comportamiento para salir bien parados en la situación en que estamos. Y esta idea debe dirigir los planteamientos pedagógicos". De esta forma, señala que: "Frente al paradigma moderno de la inteligencia como razón, y al paradigma postmoderno de la inteligencia como creatividad, los ultramodernos defendemos un paradigma ético de la inteligencia. Y consideramos que la teoría y práctica educativa deben fundarse en esta idea. Pero, además, la ultramodernidad afirma la índole social de la inteligencia, pues, aunque es una facultad individual, se desarrolla siempre en un contexto social, que la favorece o la deprime." 5

A partir de estas consideraciones, el profesor Marina establece la figura del docente, cuyos rasgos son los siguientes:

- El nuevo profesor debe concebir la educación como un proyecto ético

\footnotetext{
${ }^{3}$ HARGREAVES, Andy et al. Una educación para el cambio. Madrid: Octaedro, 1998.

${ }^{4}$ MARINA, José Antonio. "Profesores para un mundo ultramoderno". En: Cuadernos de Pedagogía. 2001. 304.

${ }^{5}$ Ibíd. P. 20.
} 
- El nuevo profesor debe ser un experto en educación

- El nuevo profesor debe entrenar para la acción

- El nuevo profesor ha de ser un experto en resolución de conflictos

- El nuevo profesor tiene que ser un experto en colaboración

- El nuevo profesor deberá adoptar un papel más activo

- El nuevo profesor debe ser un buen propagandista de la educación

Posteriormente, expone que "El nuevo Instituto Superior de Formación de Profesorado tiene que encargarse de estudiar y definir el nuevo modelo de profesor que necesitamos, que precisa la sociedad española, y poner los medios para conseguir su formación."

Debemos entender que el enfoque que nos propone el profesor Marina, se aplica a la realidad educativa española de donde es parte; sin embargo, podemos apreciar algunas relaciones con nuestra realidad educacional chilena, cuando se nos indica, desde el punto de vista constitucional y legal, que la educación es un derecho y una obligación que el estado debe garantizar. Esta visión idealista se contradice con la dura situación que enfrentan miles de estudiantes, especialmente en el sistema de Educación Superior universitario, cuando no encuentran posibilidades de pagar sus aranceles que permitan terminar sus carreras, o bien endeudar a sus padres y a ellos mismos, hipotecando tanto su presente como su futuro.

La Constitución Política actual ${ }^{7}$ (CPR) dispone dos numerales del artículo 19 para su tratamiento: los numerales $10 .^{\circ}$ y $11 .^{\circ}$, consagrando el Derecho a la educación y la libertad de enseñanza respectivamente. Sin embargo, de acuerdo con el artículo 19 numeral 10, al no gozar de garantía constitucional, prima el Derecho de los sostenedores sobre el Derecho de los ciudadanos a la educación.

El texto señala lo siguiente:

Art. 19. La Constitución asegura a todas las personas:

10. ${ }^{\circ}$ El Derecho a la educación.

La educación tiene por objeto el pleno desarrollo de la persona en las distintas etapas de su vida.

Los padres tienen el Derecho preferente y el deber de educar a sus hijos. Corresponderá al Estado otorgar especial protección al ejercicio de este Derecho.

La educación básica es obligatoria, debiendo el Estado financiar un sistema gratuito con tal objeto, destinado a asegurar el acceso a ella de toda la población.

Corresponderá al Estado, asimismo, fomentar el desarrollo de la educación en todos sus niveles; estimular la investigación científica y tecnológica, la creación artística y la protección e incremento del patrimonio cultural de la Nación.

Es deber de la comunidad contribuir al desarrollo y perfeccionamiento de la educación;

\footnotetext{
${ }^{6}$ MARINA, José Antonio. Ibíd. P. 21.

${ }^{7}$ En adelante, de forma indistinta, Constitución Política de la República o CPR.
} 
11. ${ }^{\circ}$ La libertad de enseñanza incluye el Derecho de abrir, organizar y mantener establecimientos educacionales.

La libertad de enseñanza no tiene otras limitaciones que las impuestas por la moral, las buenas costumbres, el orden público y la seguridad nacional.

La enseñanza reconocida oficialmente no podrá orientarse a propagar tendencia político partidista alguna.

Los padres tienen el Derecho de escoger el establecimiento de enseñanza para sus hijos.

Una ley orgánica constitucional establecerá los requisitos mínimos que deberán exigirse en cada uno de los niveles de la enseñanza básica y media y señalará las normas objetivas, de general aplicación, que permitan al Estado velar por su cumplimiento. Dicha ley, del mismo modo, establecer los requisitos para el reconocimiento oficial de los establecimientos educacionales de todo nivel.

De esta manera, si bien en Chile la educación es un derecho constitucionalmente establecido en el artículo 19 números 10 y 11, existe una brecha que separa el deber ser de la norma constitucional con la realidad educacional chilena. El derecho pasa a ser solo una mera declaración programática, que no tiene aplicabilidad ni eficacia, en la medida que no existe una fiscalización real de tal derecho y al no haber entes que monitoreen su estricto cumplimiento.

Podemos ver como una constante, desde la segunda mitad del siglo XX, la afirmación que la meta de la mejora en la educación es formar ciudadanos críticos y transformadores; sin embargo, nuevamente en la educación chilena esta afirmación, que aparece en las distintas reformas educacionales, no se ha cumplido, por diversos motivos. En la época actual, en que la generación de nuevas tecnologías en el campo de la información, la obsolescencia del conocimiento y el proceso de la globalización tienden a una mayor incertidumbre y más cambios en la educación, la función y el papel del profesor debe cambiar significativamente, aprovechando el conocimiento y experiencias básicas de sus alumnos y construir el conocimiento juntos, colaborativamente, pero para ello debe haber una participación de todos los actores del proceso enseñanza-aprendizaje.

La participación de los distintos actores del proceso enseñanza-aprendizaje determinará cuáles son las políticas educativas más efectivas que permitan contribuir a la mejora en la calidad de la educación y que fomenten la innovación, de esta manera el aprendizaje será concebido como un proceso permanente, que se da a lo largo de la vida del ser humano y no como algo transitorio y superficial. El Estado debe contribuir cualitativa y cuantitativamente para elaborar políticas que consideren tal participación, lo que enriquecerá las posibilidades que estos principios rectores educativos sean aplicados por la sociedad.

El profesor debe ser un guía que facilite el proceso enseñanza-aprendizaje del alumno, pero también debe cumplir una función tutorial cuando la realidad así lo amerite, entregando sus conocimientos y experiencias a sus alumnos. Además, la creatividad de los alumnos permitirá que el profesor junto con éste construya el conocimiento, haciéndolo más compartido. Una estrategia para ello, puede ser el fomento del espíritu heurístico e indagador que da la investigación como herramienta de la adquisición del conocimiento. 


\section{Los alumnos como protagonistas del proceso}

El estilo de aprendizaje se considera como "El modo personal de emplear y organizar el pensamiento y la actividad para afrontar y responder a las situaciones de aprendizaje" $"$. La misma metodología se utiliza con los demás aspectos, se preguntan y responden qué es, por qué es importante, qué aspectos me dan información, qué puedo hacer. Estos factores del aprendizaje permitirán que el profesor efectivice el proceso educativo y logre una mayor capacidad de mejora respecto de los objetivos establecidos. El estilo es un sello individual y una forma de aprender; la competencia instrumental y los conocimientos previos pasan a ser conductas de entrada; las estrategias para aprender son metodologías de aprendizaje; la motivación para aprender y el autoconcepto son percepciones subjetivas que tiene el alumno y que surgen de su propio interior como ser humano; el grupo-clase permite que el estudiante logre mayor integración, comunicación, cohesión y sentido de pertenencia, considerando los factores espaciales y temporales; el equilibro personal se da en la medida que el estudiante madure como persona, por lo que es un factor personal; el contexto escolar debe ser parte de la gestión que asumen los diversos actores; el entorno familiar es fundamental en los procesos de asunción de normas, valores y tradiciones, por parte del alumno; finalmente, el contexto social cercano pasa a ser un agente de socialización externo del estudiante que es influyente en el papel que asumirá éste en la sociedad.

En nuestro sistema educativo, los estudiantes son el reflejo de los diversos agentes de la sociedad, en este aspecto influyen principalmente la familia, la escuela, los medios masivos de comunicación, los grupos de pares. De esta manera, existe una tendencia a que sean funcionales al modelo que aspira la sociedad, esto se comprueba a través de la escasa capacidad de pensamiento crítico y la actitud pasiva frente al proceso enseñanza aprendizaje. Por otra parte, la diferencia abismante en la calidad de la educación y de los servicios entre los establecimientos públicos y privados de Enseñanza Básica y Enseñanza Media conspira con el principio de igualdad ante la ley.

Los estudiantes asumen una actitud pasiva, porque están desmotivados frente a un futuro que no ven provechoso para ellos, además están los ejemplos negativos que ofrecen algunos líderes de los sectores predominantes de la sociedad chilena, que conducen a la indiferencia y a cuestionarse hasta qué punto es valiosa la educación para ellos.

Frente a esta caracterización de este principal actor, resulta conveniente reencantarlo, proponiendo una educación que se acerque más a sus inquietudes e intereses y en la cual participen activamente en la generación del conocimiento, en el caso específico de la educación universitaria.

Por consiguiente, el alumno debe ser consciente y partícipe en un conjunto de derechos y obligaciones, que supone ser parte integral del proceso enseñanza aprendizaje.

\footnotetext{
${ }^{8}$ GARCÍA, R.J., MORENO, J.M. y TORREGO, J.C. Orientación y Tutoría en la Enseñanza Secundaria. Zaragoza: Editorial Edelvives, 1996. P. 1.
} 


\section{La cultura como factor determinante en la educación}

"Para nosotros, la cultura representa el conjunto de significados, principios, valores, creencias y prácticas compartidos por los miembros de una organización, que dan a ésta una identidad propia y determinan la conducta peculiar de los individuos que la forma y la de la propia institución." "En esta concepción de la cultura están incluidos las tres perspectivas de la cultura: antropológica-sociológica, estética y educativa; por lo tanto, resulta ser un concepto globalizador y sintetizador de la cultura.

La posición del profesor Joaquín Gairín se ve claramente reflejada en la siguiente afirmación:

Una nueva visión de la Educación Superior supondría, según la Declaración Mundial sobre la Educación Superior, avanzar en: igualdad de acceso; fortalecer la participación y promoción del acceso a las mujeres; promover el saber mediante la investigación en los ámbitos de la ciencia, el arte y las humanidades y la difusión de sus resultados; orientación a largo plazo fundada en la pertinencia a las demandas de la sociedad; reforzar la cooperación con el mundo del trabajo y el análisis y la previsión de las necesidades de la sociedad; la diversificación como medio de reforzar la igualdad de oportunidades; medios educativos innovadores que potencien el pensamiento crítico y la creatividad; y protagonismo reconocido de los profesores y estudiantes." 10

Posteriormente, añade que hay que “... reforzar los procesos de evaluación de la calidad, considerar el potencial y desafíos de la tecnología, reforzar la gestión y el financiamiento de la Educación Superior y fomentar la puesta en común de los conocimientos teórico y práctico. ${ }^{11}$

En este párrafo observamos que su visión toma aspectos esenciales de la Declaración Mundial sobre la Educación Superior, pero le añade otras propuestas como el desarrollo de la tecnología, la administración eficiente de la Educación Superior y la relación dinámica entre la teoría y práctica.

Indiscutiblemente el autor plantea un cambio estructural, que considere a los distintos actores que participan en el proceso enseñanza-aprendizaje, asumiendo los profesores un rol participativo en la conducción de la educación, es decir, como líderes de la comunidad. A partir del rol como líder del profesor, se puede mejorar y optimizar los cambios en la universidad, sin descuidar a los otros actores del sistema educativo. Por otra parte, dándole una importancia decisiva a la investigación científica, la generación de conocimiento y al avance cualitativo y cuantitativo de la Ciencia.

Gairín plantea que para modificar la cultura hay que hacerlo desde dentro, a partir de las inquietudes y necesidades, pero sin dejar de considerar las ayudas externas. Se debe analizar la cultura existente y apreciar sus debilidades y fortalezas, la necesidad del cambio producto de resultados negativos. Por lo mismo señala que: "Se entiende así el cambio

\footnotetext{
9 GAIRÍN, Joaquín. "Reconstruir la universidad a través del cambio cultural”. En: Primer Congreso Internacional "La universidad en el mundo: análisis de su trayectoria para un futuro mejor”. México D.F.: Facultad de Educación. Universidad de Anáhuac, 19-21 de octubre de 2004 (ponencia multicopiada). P. 2.

${ }^{10}$ Ibíd. P. 15.

${ }^{11}$ Ibíd.
} 
como un proceso paulatino en el que intervienen circunstancias internas y externas, que se acompañan de cambios simbólicos lo suficientemente significativos como para hacer consciente al colectivo de que la realidad está cambiando y de que emerge una nueva cultura." 12

Todo cambio en una organización supone establecer evaluaciones internas y externas que nos den un diagnóstico y un pronóstico, para luego, a partir de la información recolectada, procesada y analizada, tomar la decisión de llevar a cabo las transformaciones determinantes.

La cultura en la universidad la hacen los que viven esta institución de Educación Superior; sin embargo, éstos se ven influidos por la tradición y la cultura oficial. Por lo que, para cambiar la cultura en la universidad, es conveniente establecer consensos en las diversas instancias donde surjan conflictos, además implica reformas estructurales en que los distintos miembros de la comunidad universitaria deberán asumir roles determinantes y en las que los consensos serán situaciones decisivas en la toma de decisiones.

En el campo del Derecho, el sistema jurídico es concebido como el conjunto de elementos normativos y culturales que caracterizan en un estado a las instituciones y operadores del Derecho. Así el sistema incluye a los abogados, jueces, fiscales y funcionarios judiciales, estudiantes de Derecho; al Poder Judicial, a las prácticas y formas de hacer operativo el Derecho y las normas e instituciones jurídicas.

Para Friedman (1990) el sistema jurídico está en todas partes, con nosotros y a nuestro alrededor. Si el Derecho se identifica con el ordenamiento jurídico y éste es un mecanismo de control social, entendido como todo el entramado de normas y procesos que atribuyen consecuencias jurídicas a determinados actos de nuestra vida, el sistema jurídico es una parte del sistema de control social. El sistema jurídico involucra una estructura (el esqueleto que le da forma, como el número y clase de los tribunales de justicia, su jurisdicción y las formas de apelación, entre otras), una sustancia (las normas y los modelos de comportamiento de las personas), la cultura jurídica (actitudes de las personas frente al Derecho y al sistema jurídico, sus creencias, valores, ideas y expectativas; es decir, la cultura general), el impacto (los cambios que se produjeron frente a una decisión jurídica). Por lo que las principales funciones del sistema jurídico son el control social, la resolución de litigios, la remodelación social distributiva y el mantenimiento social considerando la continuidad y el cambio social. Todas estas preocupaciones reflejan la relación dinámica, directa y dialéctica entre las ciencias jurídicas y las ciencias sociales. ${ }^{13}$

Desde esta perspectiva, debemos concebir a la cultura jurídica como parte de un sistema normativo; por lo tanto, es un elemento que se debe incluir en el proceso de formación del abogado.

El profesor Agustín Squella plantea que:

por cultura jurídica se entiende el conjunto de valores y de actitudes relacionadas con el Derecho que prevalecen en una comunidad jurídica dada. Por lo que hay que distinguir la cultura jurídica interna y externa. La cultura jurídica externa designaría el conjunto de

\footnotetext{
${ }^{12}$ Ibíd. P. 31.

13 ÁLVAREZ UNDURRAGA, Gabriel. Curso de Investigación Jurídica. $3^{\mathrm{a}}$ edición. Santiago de Chile: Legal Publishing, 2009. P. 39.
} 
valores, actitudes y orientaciones que respecto del Derecho, y más ampliamente del sistema legal, prevalecen entre la población de una sociedad determinada. La cultura jurídica interna estaría representada por el conjunto de creencias, valores, actitudes y orientaciones que respecto del Derecho prevalecen entre nuestro medio entre aquellos individuos que de manera más directa, intensa y continuada se relacionan con ese mismo Derecho en razón de sus profesiones u oficios, a saber, legisladores, jueces, abogados, funcionarios y profesores de Derecho. ${ }^{14}$

Posteriormente, el mismo autor señala que:

El Derecho es un fenómeno cultural en cuanto se trata de algo producido por el hombre en la historia para conseguir ciertos fines, tales como paz, orden, seguridad jurídica y, en la medida que le corresponde, justicia. ${ }^{15}$

En este sentido, la cultura educativa, toma a ambas culturas jurídicas: interna y externa, para apoyar en la formación de los futuros abogados En los últimos años se ha advertido un evidente cambio, tanto en las normas e instituciones y en las prácticas, que obedecen a significativas reformas legales y a una nueva forma de creer cómo se debe aplicar y entender el derecho (más bien, un cambio cultural).

Dicho proceso se ha ido evidenciando empíricamente por diversos estudios y ha estado presente, en forma muy superficial aún, en las modificaciones de los planes de estudio de las carreras de Derecho, en especial en el perfil de egreso del Licenciado en Ciencias Jurídicas.

Nuestra propuesta apunta a fortalecer una cultura jurídica que esté más atenta a las necesidades de la sociedad chilena, por lo que dicho proceso de formación del futuro abogado debe incorporar esta nueva realidad compleja y contradictoria.

La cultura oficial y dominante es la que tiene mayor proyección en las instituciones de Educación Superior pública y esto pasa también, aunque matizada con la participación del empresariado chileno y su propio enfoque de la cultura, en las universidades privadas.

De esta forma, en las universidades tradicionales (Universidad de Chile, Universidad de Santiago, entre otras) predominan ciertas tendencias características de la universidad masiva, la institución social y el modelo burocrático. En las universidades privadas, en general, se combinan el modelo colegial con el modelo empresarial, así se observa que intentan algunas ser más reguladoras del mercado, profesionalizantes y tecnocráticas (Universidad Adolfo Ibáñez, Universidad Del Desarrollo) que otras (Universidad Alberto Hurtado, Universidad Católica Silva Henríquez).

En ambos tipos de universidades se proyecta una cultura funcional a su modelo de vida y concepción de lo que debe ser la sociedad del futuro y el rol que le cabe a los profesionales que se forman en ellas. Una cultura explícita, una cultura implícita, culturas o

14 SQUELLA, Agustín. La cultura jurídica chilena. Santiago de Chile: Corporación de Promoción Universitaria, 1992. P. 30-31.

${ }^{15}$ SQUELLA, Agustín. ¿Qué es el Derecho? Santiago de Chile: Editorial Jurídica de Chile, 2007. P. 18. 
subculturas de los actores que participan en la vida académica y movimientos culturales que se insertan en estas organizaciones.

Nuestra propuesta se centra en una cultura universitaria que considere a todos los miembros o estamentos de la vida académica; por lo tanto, requiere la participación del colectivo e insertándose en las necesidades de la sociedad chilena. Desde esta perspectiva, es fundamental reforzar las tres misiones de la universidad: docencia, investigación y extensión. En este sentido, la investigación jurídica puede entregar un significativo aporte a la cultura jurídica.

Para llevar el cambio como cambio cultural, es conveniente señalar que en la Teoría General del Derecho se alude a las fuentes materiales y las fuentes formales y, tratándose de estas últimas, encontramos a la legislación y la costumbre, quedando la doctrina de los autores y la jurisprudencia como fuentes materiales de relativa importancia. Sobre el particular, cabe advertir que en nuestro sistema jurídico concibe a los principios generales del Derecho y la equidad como fuentes formales subsidiarias de la legislación.

El sesgo positivista y normativista imperantes en nuestra comunidad jurídica hace que la principal, y más importante fuente del Derecho, sea la legislación. Ello encuentra explicaciones culturales e históricas, especialmente en la pertenencia a la familia jurídica romanista continental. Entre nosotros, la denominada estructura escalonada del orden jurídico se representa por la imagen de una pirámide: en la cúspide, una sola norma (la Constitución); bajo ésta, las leyes creadas conforme a la Constitución; bajo las leyes, las sentencias judiciales, los decretos administrativos y los actos de los particulares.

Ahondando en el mismo sentido, Agustín Squella señala que predomina en la comunidad jurídica una comprensión del Derecho en términos de una realidad específica y exclusivamente normativa, lo cual se expresa en el convencimiento ampliamente compartido -y con signos de evidente estabilidad- de que el Derecho es el conjunto de normas jurídicas vigente en un lugar y tiempo determinados ${ }^{16}$.

Lo anteriormente expuesto nos lleva a preguntarnos, partiendo de la base de que la legislación es casi la exclusiva fuente del Derecho en nuestra comunidad jurídica, ¿qué ocurre con la jurisprudencia y la doctrina?

La idea asumida culturalmente en Chile de que los jueces "son los ojos del legislador" o que son "esclavos de le ley" -al decir de Bello- no deja de representar la adhesión al positivismo normativista que se enseña tradicionalmente en las Escuelas de Derecho y que reflejan el temor de entregarle a la judicatura un poder creador de normas. Por lo menos, se advierte así en lo que son las manifestaciones explícitas de los operadores jurídicos. También se encuentra dicha posición en el plano de la doctrina. Así Agustín Squella resume la actividad jurisdiccional de la siguiente forma: "Una concepción mecanicista y logicista, que se traduce en la creencia acerca de que la aplicación del Derecho por parte de los jueces debe tener lugar sobre la base de razonamientos de tipo deductivo a partir de normas abstractas y generales de Derecho legislado" "17. Agrega el profesor Squella que: “... en la interpretación y aplicación de las leyes, (los jueces) deben proceder, siempre e invariablemente, sin apartarse un punto de éstas, no siéndoles

\footnotetext{
${ }^{16}$ SQUELLA, Agustín. La cultura jurídica chilena. Op. Cit. Pp. 30-31.

${ }^{17}$ Ibíd. P. 42.
} 
permitido llevar a cabo ninguna apreciación estimativa del texto de la ley ni de los efectos que puedan derivarse de su aplicación estricta a un caso dado"18.

Así las cosas, la conclusión podría ser que en Chile hay poco espacio para la labor creadora jurídica de parte de los jueces. No obstante, Squella también reconocía en el año 1992 y como parte de una cultura jurídica implícita aquella

práctica (consistente) en que los jueces se aparten en el hecho del texto de la ley cuando en conciencia no aprueban sus soluciones normativas o bien los resultados que se seguirían de la aplicación de éstas soluciones a un caso dado, pero que, en tal evento, opten comúnmente por no declararlo así en su fallo y reemplacen esta declaración por algún tipo de argumentación interpretativa $-\mathrm{o}$ aparentemente interpretativa- que manteniéndose formalmente vinculados a la ley, les permite, sin embargo, llegar a soluciones que aprueben en conciencia y que no coinciden estrictamente con las que han establecidos las leyes ${ }^{19}$.

Finalmente, la doctrina o estudio que hacen los tratadistas sobre las ciencias jurídicas, y con ello, de las normas, los hechos y los valores, como señala Miguel Reale ${ }^{20}$, en Chile se ha desarrollado escasamente, más como exégesis de normas jurídicas y no como ciencia.

Por lo tanto, para operativizar el cambio como cambio cultural en las escuelas de Derecho, implicaría modificaciones estructurales en el tiempo y en el espacio, en el que todos los estamentos académicos deben participar innovadoramente, sin temores, miedos y resistencias a estos cambios que la sociedad chilena lo demanda; todo esto incidiría en una nueva cultura jurídica chilena y el mejoramiento del sistema jurídico del país.

En el plano de la enseñanza del Derecho en Chile, ésta se ha caracterizado por ser discursiva, memorística y repetitiva, omitiendo la interpretación crítica y creativa que puedan tener los estudiantes, generalmente acostumbrados a un sistema educativo que privilegia la pasividad y la descripción de los fenómenos jurídicos.

La caracterización del académico e investigador del Derecho en Chile estaba acentuado por sus conocimientos profesionales, exigiéndole ser un buen operador, y en el mejor caso, como docente, un descriptor de los estatutos jurídicos que le tocaban impartir.

De esta manera, el profesor entregaba un conjunto de conocimientos y experiencias profesionales a los estudiantes, en formato de clase magistral, con escasa posibilidad que los alumnos participaran en el proceso enseñanza-aprendizaje. En su metodología de la enseñanza predominaba el método pasivo, utilizando esencialmente como técnicas la exposición de los hechos y el Derecho y, a veces, la técnica de la pregunta. No se empleaban métodos activos, salvo en casos excepcionales, ni se consideraba el aporte de las ciencias jurídicas latinoamericanas en este aspecto. La investigación jurídica, que realizaba este académico como forma de reciclar los conocimientos, prácticamente estaba reducida a

\footnotetext{
${ }^{18}$ Ibíd. P. 42.

${ }^{19}$ Ibíd. P. 43.

${ }^{20}$ REALE, Miguel. Teoría Tridimensional del Derecho. Valparaíso, Chile: Edeval, 1978.
} 
un estudio meramente descriptivo de datos obtenidos en las normas, en la escasa doctrina y en la jurisprudencia de los tribunales; lo que reiteradamente entregaba en sus clases ${ }^{21}$.

En el plano de la metodología de la enseñanza del Derecho, tanto el académico como el estudiante han continuado impulsando la tradicional clase expositiva magistral. En el caso del profesor, porque se trata de un método que le ha dado resultados históricamente, además, permite cumplir con todos los objetivos y la cantidad de contenidos establecidos en el programa y porque innovar en otros métodos activos supone mayor audacia y nuevos desafíos que implican más dedicación respecto de las estrategias que tendría que aplicar. Lo mismo acontece en la gran mayoría de los estudiantes chilenos, quienes se sienten más seguros con la aplicación de este método por parte de sus profesores, porque si se asumen metodologías más activas, deberán preparar más las clases, leer los textos asignados, analizarlos, interpretarlos críticamente; por lo tanto, como en el sistema educativo de la Enseñanza Básica y Enseñanza Media no se entregan estas competencias y habilidades, el estudiante universitario chileno tiene escasa comprensión de textos cuando se inserta en el sistema de Educación Superior universitario. Nuestro enfoque es que el objeto de estudio del Derecho no son solamente las fuentes formales del Derecho (Constitución, ley, decretos, reglamentos, ordenanzas, instrucciones, resoluciones, jurisprudencia, costumbre, actos jurídicos, actos corporativos y principios generales del Derecho), sino también las realidades sociales que definen la eficacia de las normas jurídicas. Por lo tanto, el objeto de investigación del Derecho son las normas, los hechos y los valores, los que no se dan separados, coexisten incorporados en una unidad armónica, dialéctica y concreta. Respecto de la norma, se investiga lo que los hombres dicen que hacen en el Derecho. Del hecho o conducta social se investiga lo que los hombres hacen en la vida cotidiana con el Derecho.

De la variable axiológica o valorativa se señala que tanto sobre la norma como del hecho social regulado hay valores o intereses protegidos que cruzan el discurso normativo y la realidad socio histórica. Por lo tanto, el objeto del conocimiento en las ciencias jurídicas son los hechos, las normas y los valores. El investigador jurídico estudia el mundo objetivo y real donde operan las normas.

El conocimiento jurídico es el resultado de la investigación que se ha realizado en el Derecho en el tiempo y en el espacio. Luego, las características del conocimiento jurídico son la objetividad, racionalidad, logicidad, metodicidad, sistematicidad, flexibilidad, lenguaje técnico y preciso. La función interdisciplinaria de la ciencia es un aspecto que se da en la práctica cotidiana cuando se forman equipos de investigación con profesionales de diferentes áreas del conocimiento, dentro de las cuales están los abogados, lo que permite obtener un conocimiento más integral de la realidad que pretendemos estudiar. La función transdisciplinaria consiste en entregar un aporte relevante y pertinente que cruce a las distintas disciplinas del Derecho. La función disciplinaria está dada por el valor y utilidad que tiene la disciplina en la formación del futuro abogado. Por lo que el rol de la docencia jurídica que permita cambiar la cultura para cambiar la universidad debe concentrar sus

\footnotetext{
${ }^{63}$ Esta caracterización es genérica; sin embargo, es conveniente señalar los esfuerzos por revertir esta situación por parte del enfoque surgido en la reforma universitaria de 1967 en las universidades de Chile, Católica y de Concepción, apoyadas por el programa de modernización de la enseñanza del Derecho financiada por la Fundación Ford. Al respecto, mayores datos se pueden ver en el texto de ÁLVAREZ UNDURRAGA, Gabriel. Curso de Investigación Jurídica. Santiago de Chile: Legal Publishing, 2009. Unidad III: La investigación jurídica.
} 
esfuerzos en un modelo de aprendizaje del Derecho que tome en cuenta a la investigación jurídica como un espacio que enriquezca las posibilidades del estudiante de conocer el Derecho por sí mismo, identificando, planteando y formulando el problema objeto del conocimiento jurídico, analizando e interpretando normas, doctrinas y jurisprudencia de los tribunales y buscando soluciones a estos mismos problemas. Debemos pasar del modelo de enseñanza, que prima en nuestras universidades, a un modelo de aprendizaje, en el cual se promueva e interese al alumno a pensar en el Derecho, apoyado por la incorporación progresiva de las nuevas tecnologías de información, Internet y las redes de transmisión de datos, porque en el futuro la universidad será una combinación de actividades presenciales y virtuales, ya que una significativa parte de la oferta académica se desarrollará mediante las redes de transmisión de datos y todo esto supone investigar.

\section{Los procesos de cambio en las universidades chilenas}

Las instituciones de Educación Superior del país han comenzado una discusión del modelo a partir del enfoque por competencias. Para iniciar la discusión, se elaboró un diagnóstico de la realidad de las universidades chilenas, promovidas por el Ministerio de Educación, a fines de la década de la década de 1990; posteriormente, siguiendo las propuestas de la Declaración de Bolonia, a partir de los inicios de los años 2001 y 2002, la gran mayoría delas universidades chilenas discuten cómo se insertan en este proceso de cambios, considerando sus propias especificidades. De esta forma, progresivamente las universidades tradicionales (Universidad de Chile, Universidad Católica, Universidad de Santiago, entre otras), universidades regionales (Universidad de Concepción, Universidad de Valparaíso, Universidad de Talca, entre otras) y universidades privadas (Universidad Central, Universidad Diego Portales, entre otras) han elaborado propuestas de misión y visión de la universidad, nuevas mallas curriculares, etc.

La propuesta es que este enfoque se realice considerando a los diversos estamentos académicos, directivos, administrativos, obreros, estudiantiles y de padres y representantes; tomando como base la realidad del país y sus necesidades de profesionales que permitan la transformación de la sociedad chilena.

El cambio es determinante para las instituciones de Educación Superior chilenas. Con el advenimiento de la transición a la sociedad democrática, después de 17 años de gobierno militar y universidades intervenidas y funcionales al régimen, la sociedad civil organizada y el movimiento estudiantil presionó fuertemente para generar modificaciones, éstas se dan cualitativa y cuantitativamente en forma paulatina, porque no se rompe del todo con las herencias del pasado: Constitución de 1980, modelo económico de mercado, privatización de la educación y la salud, fomento al individualismo y lentitud en pagar la deuda social y las violaciones a los Derechos humanos, especialmente con los sectores más postergados de la sociedad.

La universidad durante el régimen militar deja de ser pública, el Estado sólo subsidiariamente asume su rol de protección social, por otra parte, antes de entregar el mando, las autoridades del gobierno militar aprueban la LOCE. 
Esta situación se ha mantenido históricamente en estos veinte y un años, obligando a vastos sectores sociales desprotegidos a endeudarse para acceder a la educación universitaria. El financiamiento de las universidades del Consejo de Rectores, que reciben mayor aporte del Estado, es escaso y las posibilidades de becas a los estudiantes provenientes de los sectores más desasistidos de la sociedad chilena es limitado. Por todas estas consideraciones es un imperativo el cambio en estas instituciones. Sin embargo, cualquier propuesta de cambio debe considerar cómo promoverlos y cómo disminuir las resistencias. Luego, todo cambio supone consensuar las propuestas, en esta etapa del proceso que la sociedad chilena todavía está viviendo. Por lo tanto, estamos refiriéndonos a un cambio estructural de roles y relaciones entre los distintos sectores comprometidos. Lógicamente se producirán resistencias al cambio y a la innovación durante este proceso, que es controversial, dinámico y dialéctico, pero para ello habrá que requerir de un clima organizacional y cultura organizacional armónicos con el cambio propuesto.

Indiscutiblemente en el caso chileno, la transición hacia la democracia, las legítimas aspiraciones del pueblo chileno represadas por el régimen militar y el surgimiento de los movimientos sociales y políticos generan posibilidades de cambio en las distintas áreas de la sociedad chilena. La educación no está ajena a este proceso de cambios, por lo mismo, considerando la globalización y la obsolescencia del conocimiento, el Estado chileno suscribe y ratifica una serie de tratados internacionales, uno de los más significativos fue con la Unión Europea. En el marco del tratado de Chile con la Unión Europea está, como uno de los aportes en materia universitaria, la armonización de los títulos y grados profesionales. A partir de esta nueva obligación jurídica, las universidades chilenas inician el proceso de reformas. Por lo tanto, la propuesta de utilizar las cinco fases del modelo de estudio de las instituciones de Educación Superior: información, contextualización, actuación, cambio y difusión, es plenamente aplicable a la Educación Superior chilena, por supuesto, hay que adaptarlo a cada modelo universitario y considerar la idiosincrasia de los actores involucrados.

Si la evaluación externa considera el proceso de evaluación interna de la institución, puede ayudar a la mejora y los cambios. Toda evaluación externa debe realizarse por organismos serios, probos, legítimos, capacitados y objetivos. Esta situación en el caso chileno no ha sido muy claro en algunos casos de procesos de acreditación (un ejemplo de esto se evidenció en la acreditación de la Universidad de Las Américas). Por otra parte, si proponemos evaluaciones externas, éstas deben ser completas, estudiando y evaluando todos los factores del proceso, y generar participación de todos los actores de la institución evaluada. Deben acceder a todos los que son parte integral de la institución, para obtener información confiable y válida.

\section{Estadios de desarrollo organizativos}

En un hecho, ampliamente reconocido por la comunidad universitaria, que la gran mayoría de los profesores que componen el estamento académico de las distintas escuelas de Derecho de las universidades chilenas está constituido por abogados sin formación pedagógica, algunos han asistido a cursos de capacitación docente impartidos por las universidades, otros agregan sus títulos de Magíster de especialización en áreas del 
Derecho y, los menos, son Doctores en Derecho. Por esta razón, considerando las estructuras de los recursos humanos que interactúan, podemos decir que el rol del docente se asume como impartiendo una clase expositiva, memorística, libresca, verticalista, y el alumno, a su vez, asume una actitud pasiva frente al proceso enseñanza-aprendizaje, debido también a que es más cómodo para él no comprometerse con asignaciones de lecturas ni participar en el proceso.

En general, la visión de la organización como marco, es propia del enfoque científico-racional, porque así lo es la Ciencia Jurídica. Predomina una perspectiva legalista del Derecho positivo, con escasa posibilidad de contextualizar con otras áreas de las ciencias sociales y reducida vinculación de la norma con la sociedad; sin embargo, hay esfuerzos porque tal situación cambie y se pase al segundo estadio o nivel de desarrollo de la organización como contexto. En este sentido, las Facultades de Derecho tradicionales (Universidad de Chile y Universidad Católica) y algunas privadas (Universidad Diego Portales y Universidad Central) han realizado cambios en sus mallas curriculares a partir del nuevo enfoque en el proceso de formación del futuro abogado.

El paradigma educativo pasa a ser fuertemente tecnológico, porque se concibe formar a un abogado operador de las normas jurídicas, altamente profesionalizante en sus actividades laborales, se busca un producto que se inserte en las demandas propias del mercado de trabajo, la tipología profesional es altamente técnico, que conozca la legislación positiva y la aplique en la resolución de los problemas. El modelo de formación es cultural y de competencias. Sin embargo, es conveniente precisar que esta perspectiva debe matizarse por el grado de complejidad del proceso en las organizaciones educativas y la dinámica de los cambios influidos por la globalización y el desarrollo acelerado de los conocimientos, lo que determinan transformaciones que se están produciendo en las mallas curriculares de las escuelas de Derecho de este país.

Indiscutiblemente se debe pasar al segundo estadio, la organización como contexto, en la medida que se produzca la internalización de la nueva cultura jurídica y cómo ésta afecta a la formación de los abogados. Para que se produzca esta nueva situación, la propuesta básica consiste en la participación de todos los estamentos en la discusión y abrir espacios para establecer modificaciones de planes, programas y políticas en materia de formación de abogados en el país.

\section{Evaluación institucional}

Si entendemos que cada universidad chilena tiene su misión y visión sobre el quehacer para formar profesionales que se insertan como sujetos de cambio en la sociedad, además, objetivos a largo, mediano y corto plazo, también recursos humanos diversos y variados, podemos inferir que se deben establecer vínculos entre objetivos, estructura y sistema relacional que no siempre son lógicos y coherentes. Por otra parte están las concepciones e ideologías imperantes, que pueden ser contradictorias y conflictivas. Todos los aspectos apuntados nos demuestran que el proceso de evaluación debe ser asumido con las características indicadas, tomando en cuenta el marco nacional y el marco internacional, el desarrollo acelerado del conocimiento científico, la informática y la globalización. En este sentido, concordamos con el enfoque holístico en la materia. 
Si la perspectiva cultural enfatiza en su valor como instrumento para comprender una realidad determinada, en este sentido la evaluación servirá como un medio para intercambiar puntos de vista, intercambiar criterios y participar en la elaboración de propuestas; y la perspectiva socio-crítica enfatiza en la naturaleza política de las instituciones, considerándolas como estructuras de poder, y en su capacidad para promover y transformar la realidad, en este enfoque la evaluación debe evidenciar las disfunciones existentes e iniciar el proceso de cambio que mejore el valor y sentido social de la organización.

En la realidad de la Educación Superior chilena ambas perspectivas nos sirven para tener una evaluación más integral, totalizadora, completa, participativa y dinámica acerca cómo operan estas organizaciones. De esta manera, la evaluación implicará realizar un diagnóstico, establecer un pronóstico de la institución y finalmente, entregar una propuesta armónica con los datos analizados.

La autoevaluación, bien aplicada, es un potente instrumento de caracterización de la conducta asumida por los diversos actores de la universidad chilena. Por lo mismo, debe realizarse en forma objetiva, sistemática, permanentemente si se quieren introducir cambios significativos. Esta autoevaluación de los procesos educativos debe ir acompañada de la evaluación interna y de la evaluación externa; por lo tanto, las tres permitirán una información más confiable y válida que permita mejorar el proceso enseñanza-aprendizaje. Sin embargo, es conveniente resaltar que la institución debe ser lo suficientemente democrática, autónoma, considerar las diferencias individuales y fomentar el espíritu colectivo para llevar a cabo la autoevaluación, respetando sus resultados.

Toda evaluación profunda debe buscar las causas que generan determinados problemas que ameritan ser resueltos y también los posibles efectos que se pueden dar. En este sentido conviene que los procesos evaluativos deben ser unitarios, integrados, analíticos y sistémicos. Si evalúo en forma profunda y exhaustiva a una institución universitaria chilena, primero, debo identificar sus fortalezas y debilidades, a partir de estas últimas debo analizar el diagnóstico del problema, posteriormente, visualizar el pronóstico, ambos aspectos entregarán las causas y efectos del problema objeto de estudio y con ello entregaremos una propuesta de mejora del proceso.

\section{La calidad en la educación universitaria}

Durante el período del régimen militar, la concepción de calidad en la educación que predominaba era funcional a los objetivos y metas del Gobierno e influida por el modelo neoliberal imperante. Posterior al advenimiento de la transición a la democracia, se produce una mayor apertura a concepciones menos rígidas, pero sin abandonar este modelo, por tanto, cónsonos con la idea de identificar calidad con eficacia. Durante los cuatro Gobiernos de la Concertación (1990-2010) los enfoques de la calidad fueron mutando, dependiendo de las ideas prevalecientes, así durante el período 1990-2000 los documentos de organizaciones europeas (OCDE 1991) habían proyectado sus ideas en el Ministerio de Educación de Chile, por otra parte, también se trabajó con la concepción de calidad total, muy influido por las tendencias derivadas de las ciencias administrativas empresariales; 
posteriormente, como consecuencia de la política de los mercados internacionales abiertos y la suscripción de tratados de libre comercio, especialmente con la Unión Europea, la concepción de calidad educativa se acerca a lo establecido en la Declaración de Bolonia, adaptando algunos aspectos significativos.

En todo caso, la concepción de calidad educacional que más se ajusta a la universidad chilena debe considerar los objetivos, los resultados y las causas de la eficacia de los estudiantes universitarios chilenos, por lo que la discusión sobre el tema está totalmente abierta.

La concepción neoliberal de calidad educativa no puede darse en las instituciones de Educación Superior públicas, porque tienen un marcado sesgo privatizadora, influida por concepciones empresariales que tienden a identificar a la universidad a una institución de carácter lucrativa que se maneja con las técnicas y principios propios del mercado. La concepción del Estado bienestar o asistencialista tienen un enfoque opuesto al modelo anterior y en la sociedad chilena todavía tienen sus partidarios.

Esto determina un debate en las universidades tradicionales, en las que los diversos estamentos plantean sus posiciones que, mayoritariamente, no son favorables al modelo de mercado. Sin embargo, es conveniente señalar que esta posición ha ganado adeptos en algunas universidades empresariales y corporativas (Universidad Adolfo Ibáñez, Universidad del Desarrollo, entre otras).

Al investigar más sobre la problemática de la calidad educativa en las universidades tradicionales, apoyado por el financiamiento del Estado y las ONG, se puede aproximar a un concepto más social y humanista, menos cercano a las concepciones neoliberales, con propuestas creativas que ayuden a la mejora y al cambio en los procesos educativos. Por lo mismo, las universidades deben generar espacios de discusión y líneas de investigación sobre la materia y la metodología debe armonizar con su objeto de estudio.

Los resultados obtenidos en las investigaciones derivadas de la calidad educativa deben utilizarse para comprender y mejorar la realidad, identificar las fortalezas y debilidades, para potenciar las primeras y corregir las segundas. Por lo tanto, sirven para tomar decisiones determinantes en el proceso educativo, asumiendo un modelo de calidad más justo, solidario y humano.

\section{Comunidades formativas}

Las instituciones de Educación Superior del país deben considerar el contexto institucional como espacio de innovación y de cambio, porque sus realidades internas y las externas han sufrido transformaciones que afectan aspectos determinantes de su vida académica. De la actitud individualista, propia de la sociedad que tiene un modelo neoliberal, con rasgos competitivos, privatizador, tecnócrata, autoritario, verticalista y altamente jerarquizado, se debe pasar a una actitud colectiva, propia de un Estado más humanista, con rasgos solidarios, colaborativos, horizontal, participativo. Estas actitudes se tienen que traspasar al proceso enseñanza-aprendizaje, en el marco de reformas e innovaciones institucionales, que legitimen y legalicen este proceso de cambios. Para llevar 
a cabo esta propuesta, es necesario que toda la comunidad universitaria participe activamente en la toma de decisiones, la que los afectará en su vida académica.

El profesor universitario puede asumir un papel protagónico en las innovaciones educativas en la medida que toma conciencia de sus fortalezas y debilidades, de esta manera puede potenciar sus fortalezas y corregir sus debilidades; pero también debe reconocer el rol de líder de la comunidad que tiene como docente. Además, puede participar con sus pares desarrollando una cultura colaborativa a través de diálogos, compartir experiencias didácticas y profesionales, escuchar más los problemas de aprendizaje y las necesidades de los alumnos, retroalimentándose de los procesos de autoevaluación, coevaluación, evaluación interna y externa, asociando en el proceso enseñanza-aprendizaje la teoría con la práctica, estudiando e investigando permanentemente en su disciplina, generando líneas de investigación que pueden ser aprovechadas por la institución o por la sociedad.

La cultura del cambio es un proceso que se ha dado lentamente en la evolución histórica en Chile, porque cuesta modificar instituciones tradicionales debido a una idiosincrasia conservadora, especialmente consagrada en los sectores dominantes, con un alto temor y resistencia a las transformaciones sociales, políticas, económicas, jurídicas, culturales, y por supuesto, educacionales. Los ejemplos más contundentes de esta afirmación lo tenemos en la época del Gobierno del presidente Salvador Allende (19701973), cuando distintos proyectos de transformaciones políticas, económicas y sociales fueron duramente combatidos por la oposición ligada a intereses económicos de los sectores dominantes de la sociedad y de las transnacionales. En ese período se intentó crear el proyecto de la Escuela Nacional Unificada (ENU), basada en el programa de la UNESCO sobre educación permanente; sin embargo, la oposición inició una campaña sistemática en los medios de comunicación de masas, denunciando el proyecto como atentatorio a las ideas de los chilenos y a la libertad de enseñanza. Durante el período de la administración de Eduardo Frei Ruiz Tagle (1994-2000) el Gobierno intentó aprobar un proyecto de educación sexual en los colegios del país, denominado las JOCAS (Jornadas de Conversación Sobre Afectividad y Sexualidad), la oposición de centro derecha se negó a discutirlo, porque atentaba contra la familia.

Con estos ejemplos, tratamos de establecer la dificultad de generar cambios sin un consenso que abarque a la totalidad de los miembros de una comunidad, entregándoles el máximo de información para una toma de decisión adecuada. La operatividad de los cambios para innovaciones debe ser ampliamente difundida, señalando sus ventajas y desventajas, para que la comunidad educativa tome decisiones que favorezcan las mejoras cualitativas y cuantitativas.

La universidad es un centro de educación que es universalista, como su nombre lo indica, por lo que debe estar abierta a todas las corrientes, concepciones e ideas prevalecientes en la sociedad; por lo tanto, toda universidad debe ser pluralista en sus concepciones y aprovechar la riqueza del aporte de las ideas en la discusión y el debate.

Las tres concepciones paradigmáticas: científico-racional, interpretativo-simbólico y socio-crítico son importantes, porque entregan aportes para la universidad como centro de excelencia académica y ayudan en la reflexión del tipo de profesional que se quiere formar. Si tomamos como ejemplo las Ciencias Jurídicas, en éstas prevalece el positivismo en la 
enseñanza del Derecho, sin embargo, la interpretación de las normas jurídicas, los hechos y los valores también ocupan lugar en el proceso formativo de los futuros abogados. La visión socio-crítica también, poco a poco, se está haciendo presente, a través de la investigación socio-jurídica, en la que la transformación de las instituciones y normas jurídicas pasan a ser vitales. Por otra parte, desde la visión de la organización, las perspectivas unitarias, pluralistas y dialécticas se observan nítidamente y coexisten en la formación jurídica, por las características que tiene el Derecho como Ciencia. Finalmente, respecto de sus consecuencias, el enfoque científico-racional se preocupa por definir las metas, establecer parámetros de evaluación de la docencia que permitan la calidad e innovación del proceso enseñanza-aprendizaje en función de estándares preestablecidos. El enfoque interpretativo-simbólico observa como consecuencias la preocupación por la cultura organizativa, expresada especialmente en las universidades tradicionales y de mayor prestigio, donde estudiar y enseñar, por ejemplo, en la Facultad de Derecho de la Universidad de Chile da estatus, porque allí han estudiado algunos Presidentes de la República y han hecho clases en sus aulas personalidades influyentes en la historia política, social y jurídica de Chile; por lo tanto, se manifiesta la calidad como valor percibido, la innovación y el cambio como excusas para el debate e intercambio de ideas. El enfoque socio-crítico considera el conflicto y la negociación, que son parte integral de las universidades, existe un compromiso con el cambio organizativo, la calidad es el resultado de las demandas estudiantiles y académicas, la innovación es el efecto derivado de la resolución de problemas complejos y de disfunciones de la institución universitaria. De todas maneras, es conveniente matizar la contextualización expresada, porque las concepciones se combinan generalmente y coexisten entre sí.

\section{Las tutorías académicas}

El modelo de tutoría, que debería ser aplicado en Chile, pasa por una actividad académica bien remunerada, por lo que el académico tiene que poseer altos niveles de capacitación en su cargo, además de Magíster y Doctorado en el área de la Educación. También este profesor e investigador debe producir al menos un artículo relevante en su área al año, publicado en revistas especializadas de reconocido prestigio nacional e internacional. El profesor tutor debe tener estabilidad laboral, incentivos para cumplir la función tutorial, reconocimiento en generar líneas de investigación. La institucionalidad universitaria, expresada en sus diversos actores, debe garantizar su actividad autónoma, pero eficiente.

Se trata de un imperativo disponer de profesores tutores que guíen y orienten el proceso enseñanza-aprendizaje de sus alumnos, dotándolos de más motivación en la realización de sus actividades. Esta necesidad se acentúa, debido a las fallas estructurales en la Educación Básica y Educación Media en nuestro país, por lo que la inserción de estos estudiantes en el sistema universitario resulta compleja. Frente a la necesidad de orientadores, facilitadores, en la universidad, se está tratando de profesionalizar esta función, un tanto mirada en menos tradicionalmente, pero que ahora resulta conveniente integrarla como parte de la comunidad formativa. 
Es conveniente resaltar que la universidad tiene como misión: docencia, investigación y extensión. Por lo tanto, si la actividad tutorial es parte de la función de profesor, existe una relación que vincula la actividad académica con la investigación que consiste en la generación del conocimiento. No se puede impartir docencia sin reciclar los conocimientos permanentemente y esto se logra a través de la investigación; por lo tanto, ambas deben estar unidas indisolublemente.

Respecto a la tutoría académica, el docente que va a cumplir tal función debe conocer y comprehender la realidad del sistema universitario, estar al tanto de las nuevas metodologías en los procesos de enseñanza-aprendizaje, de tal manera que la investigación resulta una necesidad ineludible que deberá cumplir para conocer a los actores, principalmente a los estudiantes.

Se puede legitimar la función tutorial en las universidades chilenas informando de la concepción integral de sus roles, así como de las necesidades que tiene el sistema universitario de tener un profesional que conozca más a fondo la realidad de la Educación Superior chilena. La toma de conciencia de la importancia del tutor, en la comunidad universitaria, va a ser lenta pero progresiva. Las universidades deben legalizar y legitimar el papel, dotándolo de mayores funciones, atribuciones y poderes.

\section{La investigación jurídica y su aporte al mejoramiento del proceso enseñanza del derecho}

La investigación jurídica se puede transformar en un potente instrumento en el mejoramiento del proceso enseñanza-aprendizaje del Derecho, si las Facultades de Derecho de las diversas instituciones de Educación Superior del país asumen que la investigación es una de las misiones más importantes y relevantes de la universidad. Mucho se ha planteado, como afirmación, que "sin investigación no hay docencia", pero la realidad de nuestras universidades en Latinoamérica, en general, y en Chile, en particular, confirma que no se cumple este enunciado, porque en la gran mayoría de los casos. Toda investigación se sostiene con recursos económicos que el Estado entrega en forma reducida y las empresas privadas simplemente no lo consideran de su prioridad. Por esta consideración de carácter económica, resulta difícil la tarea del académico e investigador la realización de un estudio, incluso en el campo de las Ciencias Sociales, específicamente en las Ciencias Jurídicas. Además, en el Derecho hasta hace pocos años, las mallas curriculares no consideraban cursos de Metodología de la Investigación Jurídica, por lo que la formación de los futuros abogados carecía de esta disciplina transversal. A nivel de postgrados, recientemente en los diversos Magíster en Derecho, con sus diversas especialidades, se considera solo parcialmente un curso que oriente a los alumnos a la elaboración de su tesis. Incluso, se ha discutido si es mejor trabajos alternativos a la tesis, con lo cual nuevamente la investigación queda relegada a un segundo plano.

La investigación jurídica, si el docente quiere utilizarla como un medio de adquisición de conocimientos del Derecho, puede convertirse en una fase activa del proceso enseñanza-aprendizaje del estudiante, quien por sí mismo, orientado por el profesor, buscará en las fuentes formales y materiales del Derecho, en las primarias, secundarias y terciarias; en las directas e indirectas; en las generales y especializadas; en las clásicas y 
actualizadas, la información y el conocimiento relevante y más significativo para él, lo que será más determinante para él en la medida que fue obtenido por su propio esfuerzo.

\section{Conclusiones}

El desarrollo acelerado de las nuevas tecnologías de información ha cambiado el modo de pensar y recrear el conocimiento científico y la producción y adquisición del conocimiento, a través de la investigación científica. La educación y los sistemas educativos no están ajenos a esta situación, que afecta principalmente a los sujetos del proceso enseñanza-aprendizaje. Desde esta perspectiva urge generar nuevos enfoques y políticas que se adapten al nuevo marco que estamos viviendo. Indiscutiblemente que en sociedades en vías de desarrollo, como la chilena, los cambios se producen lentamente, la participación de todos los actores es difícil, mas no imposible.

En nuestro país, se debe asumir el problema y enfrentarlo adecuadamente, especialmente la alfabetización multimodal con un criterio que abarque las diferentes realidades sociales, económicas, étnicas, de género, etarias, etc. En una información destacada por El Mercurio, de Santiago de Chile22, se señala que el 52\% de los 9.260 establecimientos municipales, subvencionados y privados, que participaron en la evaluación del primer censo de informática educativa efectuada por el Ministerio de Educación, no aprovecha la infraestructura informática y de software que tiene para enseñar a sus alumnos. Esto nos demuestra que falta incorporar, de hecho y de Derecho, la tecnología como un instrumento que facilite el proceso enseñanza-aprendizaje tanto para los profesores como para los alumnos. Por lo tanto, el desafío está abierto.

La educación, con sus diversos actores, está en pleno proceso de evolución considerando el marco internacional donde se insertan los sistemas educativos. Por lo que el profesor debe estar muy atento a estas transformaciones y a la complejidad del proceso enseñanza-aprendizaje, lo que influirá en su nuevo rol en la sociedad del conocimiento y la información.

Los actores del proceso enseñanza-aprendizaje deben participar activamente con propuestas novedosas que permitan transformaciones cualitativas y cuantitativas en el sistema educativo. Sin embargo, consideramos que si no se asume la transformación de la estructura de la sociedad y su modelo económico, social, político, jurídico imperante, resulta difícil sostener solo reformas cosméticas que no apuntan a soluciones de fondo sino de forma.

La violencia escolar, el absentismo, la disrupción, el abandono escolar y otros, como el trabajo infantil, son aspectos característicos de una sociedad que manifiesta grandes males sociales. Los movimientos sociales que se han dado en Chile durante el año 2011 demuestran que existe un gran descontento por un modelo impuesto por la dictadura militar y que se ha mantenido por los políticos de ambos sectores. En el plano educativo existe un segmento de la población juvenil que está excluida de las posibilidades de acceder a un futuro mejor, al tener que pagar costos considerables que hipotecan dicho futuro, Estos son aspectos que debemos reflexionar y que nos dejan los documentos estudiados en este trabajo. 


\section{Referencias bibliográficas}

ÁLVAREZ UNDURRAGA, Gabriel. Curso de Investigación Jurídica. 3. a ed. Santiago de Chile: Legal Publishing, 2009.

BAUTISTA GARCÍA-VERA, Antonio. "Alfabetización tecnológica multimodal e intercultural". En: Revista de Educación. 2007, núm. 143.

ELGUEDA, Pamela y SOLORZA, Carlos. "El 52\% de los colegios de Chile no usa la tecnología para enseñar." En: El Mercurio, lunes 30 de mayo 2011, Cuerpo A.

GAIRÍN, Joaquín. "Reconstruir la universidad a través del cambio cultural". En: Primer Congreso Internacional "La universidad en el mundo: análisis de su trayectoria para un futuro mejor". México D.F.: Facultad de Educación. Universidad de Anáhuac, 19-21 de octubre de 2004 (ponencia multicopiada).

GARCÍA, R.J., MORENO, J.M. y TORREGO, J.C. Orientación y Tutoría en la Enseñanza Secundaria. Zaragoza: Editorial Edelvives, 1996.

HARGREAVES, Andy et al. Una educación para el cambio. Madrid: Octaedro, 1998.

MARINA, José Antonio. "Profesores para un mundo ultramoderno". En: Cuadernos de Pedagogía. 2001, núm. 304.

REALE, Miguel. Teoría Tridimensional del Derecho. Valparaíso: Edeval, 1978.

SQUELLA, Agustín. La cultura jurídica chilena. Santiago de Chile: Corporación de Promoción Universitaria, 1992.

SQUELLA, Agustín. ¿Qué es el Derecho? Santiago de Chile: Editorial Jurídica de Chile, 2007. 